\title{
La batteria Valdilocchi alla Spezia, rilievo digitale e documentazione di un'architettura alla fine di un'epoca
}

The "Valdilocchi Battery" in La Spezia, digital survey and documentation of an architecture on the edge of an epoch

\author{
Giorgio Verdiani ${ }^{\text {a }}$, Ludovica Marinaro ${ }^{\text {b }}$, Denise Reitano ${ }^{c}$ \\ a Dipartimento di Architettura - Università degli Studi di Firenze, Florence, Italy, giorgio.verdiani@unifi.it \\ ${ }^{\mathrm{b}}$ Dipartimento di Scienze e Tecnologie Agro-Alimentari - Università di Bologna, Bologna, Italy, \\ ludovica.marinaro@gmail.com \\ ${ }^{\mathrm{c}}$ Landscape Architect, La Spezia, Italy, denise.reitano@yahoo.it
}

\begin{abstract}
All the fortifications have their time. They respond to specific way of defending and attacking they change in time accordingly to the terrible habits of the weaponry and technology evolution. For a long time, from the age of the great fortifications, to the use of cannons, to the invention of the aerial war, the military built heritage have seen a long transformation made of sudden architectural changes, until the moment when the possibility of bombing and attacking from the sky signed the sunset of the large over ground buildings in favour of mimetic and underground structures. The "Valdilocchi Battery" belong to the last age of the large fortress, capable to resist to ground attack while showing the aspect of an inexpugnable stronghold. Sited on the top of a hill in the eastern part of the La Spezia gulf, this battery had the function of second line defense to control the neighboring valleys. The particular pentagonal plant and the state of excellent preservation, except for a part of collapse on the front of the gorge due to the German bombing, make it an artifact of great value.
\end{abstract}

Keywords: Digital survey, World War One, architecture, Liguria, landscape transformation, lost heritage.

\section{Introduzione}

Sin dalla prima edizione della conferenza FORTMED, l'insieme di ricerche di cui questo contributo sulla Batteria Valdilocchi prosegue le attività (Di Grazia, Marinaro, 2015; Marinaro, Grazia, 2016; Marinaro, Di Grazia 2017; Marinaro, et al., 2017; Marinaro, et al., 2018), ha portato all'attenzione della comunità scientifica il cospicuo patrimonio di architettura militare del Golfo della Spezia e il suo grande valore paesaggistico, poiché costituisce un sistema di difesa territoriale unico a livello europeo per complessità e integrità. Molte strutture appaiono in questo scenario come palesi ed evidenti, per altre, una serie di sfortunati eventi hanno isolato dall'accesso e anche dalla vista la quasi totalità del manufatto. L'interesse specifico per la Batteria Valdilocchi, di fatto "scomparsa" per decenni, fa seguito al processo di alienazione della proprietà demaniale verso i Comuni del Golfo, letto più volte nel suo doppio potenziale di opportunità e minaccia, che ha visto questa fortificazione entrare nelle disponibilità del Comune della Spezia il quale ne ha programmato la conversione ad uso pubblico. 


\subsection{La batteria Valdilocchi nella storia mili- tare del golfo}

Si può meglio comprendere il ruolo della batteria Valdilocchi solo qualora la si ponga in relazione con l'intero sistema difensivo dell' arsenale marittimo militare. La Batteria Valdilocchi infatti non è che un piccolo tassello del complesso sistema di fortificazioni del golfo della Spezia. Patrimonio architettonico militare unico per estensione a livello mondiale realizzato prevalentemente nel periodo che va dall'ascesa di Napoleone Bonaparte sino alla Seconda Guerra Mondiale, esso integra fortificazioni e castelli di epoche precedenti con architetture moderne per la difesa ravvicinata della più potente base navale italiana e del polo industriale sorto accanto ad essa. Il sistema difensivo si componeva di due fronti di fortificazioni: un fronte mare, contro gli attacchi provenienti dal golfo da parte di unità navali e un fronte terra, contro attacchi condotti da truppe sbarcate in Versilia, provenienti dalla pianura Padana o dal genovese. Il fronte terra vedeva poderose opere in grado di sbarrare le vie di accesso terrestri e di battere con il loro fuoco tutte le aree circostanti la piana della Spezia. Esso era suddiviso in una rete di fortificazioni staccate, avanzate e indipendenti come prima linea, una cinta continua di sicurezza per la protezione ravvicinata come seconda linea ed una cinta di mura perimetrali armate intorno alla città come difesa estrema (Marinaro, Falqui, Reitano, 2018). Fra la Prima e la Seconda Guerra Mondiale si assistette ad una seconda fase fortificatoria, che incrementò il numero delle opere militari e modificò l'assetto di alcune di quelle esistenti per prepararle alla difesa contraerea.

Fra queste ultime vi è la batteria di Valdilocchi, la quale si inserisce nel fronte orientale del golfo ed appartiene al fronte terra che va dal monte Valeriano al monte Rocchetta. Essa fu una delle prime opere realizzate (1880) con lo scopo di impedire l'ingresso al golfo dai valichi verso la piana della Magra qualora lo sbarramento dei forti e delle batterie staccate fosse stato superato. Costruita sulla cima di un modesto rilievo, la sua posizione arretrata ma dominante le permetteva di battere l'intera piana di Spezia ed i suoi accessi orientali.
Come tutte le batterie, questa struttura era dotata di artiglieria monocalibra e monotipo, disposta su un'unica linea di fronte. Valdilocchi incrociava il fuoco con le batterie dei Cappuccini e del Molino a vento verso il valico del Termo e del Muggiano.

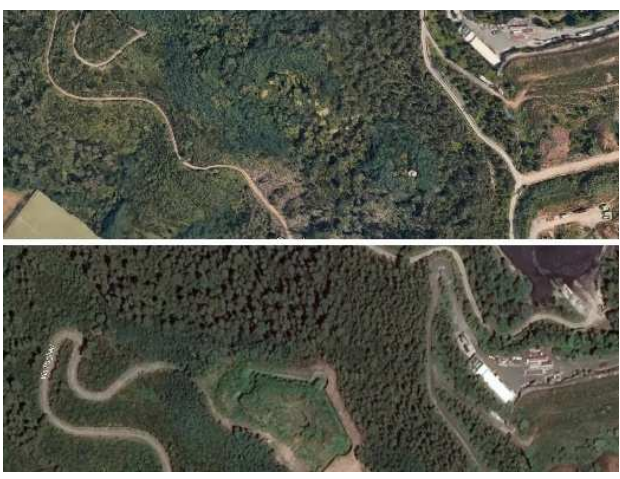

Fig. 1. Vista satellitare della Batteria Valdilocchi prima e dopo le operazioni di pulizia da vegetazione infestante. Primavera-Autunno 2019 (Google Maps and Microsoft Bing, 2019).

L'opera, pur essendo di seconda linea, fu dotata di un consistente armamento data la sua posizione a margine dell'ampio distretto logistico militare e industriale comprendente le località Valdilocchi, Pagliari, Ferrarezzola e Vallegrande dove furono installate numerose realtà industriali e militari strategiche. Come documentato dai materiali di archivio e come riscontrabile dalla recente ispezione dell'area, la batteria, a pianta pentagonale aveva un fronte principale articolato su due semi-batterie, inclinate tra loro di $130^{\circ}$ ed orientate a nord e nord-est, ciascuna con due piazzole doppie in muratura separate da traverse. Una quinta piazzola doppia era posta sul fianco sinistro rispetto all'ingresso.

L'ingresso si apriva nella cinta di gola a sinistra del punto mediano, con ponte levatoio a contrappesi su un fosso asciutto continuo di tipo "diamante" che girava attorno a tutta la struttura, protetto da tre mezze caponiere ed una caponiera ordinaria sul saliente centrale che battevano d'infilata tutti i rami del fosso.

All'interno del forte, a sinistra dell'ingresso, si trovavano il Corpo di guardia e gli alloggi degli ufficiali, mentre sulla destra gli alloggi della 
truppa; da questi si raggiungevano le gallerie di scarpa che conducevano alle caponiere e formavano un intercapedine di isolamento fungendo al contempo da galleria di illuminazione per i magazzini delle polveri.

Il cortile era il cuore della batteria; lì si affacciano vari depositi e magazzini, la cucina, i locali igienici ed un locale a pianta circolare, sotto il quale era stata ricavata una cisterna di raccolta dell'acqua piovana, che conduceva alla caponiera occidentale e all'intercapedine di ventilazione e illuminazione sotto la semi-batteria di sinistra. Sul cortile affacciano anche i corridoi di accesso alle caponiere orientali e ai depositi di polvere posti all'estremità destra della gola rispetto all'ingresso, in posizione defilata e protetta rispetto al tiro nemico, mantenuti asciutti e ventilati da intercapedini su due lati.

I laboratori di confezionamento delle cariche e dei proietti ed il magazzino dei proietti carichi erano posizionati sotto la semi-batteria di destra.

\subsection{La Batteria oggi: ai margini della vita della città}

Da ricondurre al più probabile toponimo "Valdilochi", l'etimologia suggerisce un legame con il latino locus, nel senso esteso di luogo abitato da una famiglia, ma anche di podere, di proprietà. In buona sostanza "Valdilocchi" era una valle abitata, presidiata e coltivata. è forse da imputare alla consueta ironia attribuita al destino il fatto che oggi non soltanto questa batteria, ma tutta la collina sia stata completamente dimenticata, uscendo in maniera progressiva dal circuito dei luoghi abitati e vissuti della costa orientale.

Un bosco pioniero di pini e robinie ha preso il posto dei campi e i caratteri del paesaggio rurale un tempo distintivi, sono stati sostituiti dai tratti sbozzati ed incerti del paesaggio urbano della periferia industriale e produttiva.

La costruzione dell'Arsenale Marittimo Militare, e di tutto il sistema di difesa a suo corredo, determinò anche la crescita urbana e demografica della città di Spezia che nel secolo XX conobbe un periodo di grande espansione portando alla connessione diretta con molti piccoli paesi (borgate) che punteggiavano il golfo, che divennero quartieri.
A questo processo si lega quello della crescita del polo industriale e del porto commerciale, insieme anche al comparto della cantieristica, funzioni che ancora oggi coesistono nello spazio ristretto della piana e dell'arco costiero. A valle di queste radicali trasformazioni del territorio, la collina su cui si erge la batteria oggi è saldamente stretta nell'inviluppo di infrastrutture e recinti di un'area di frangia urbana a vocazione mista industriale, portuale e produttiva.

Questa destinazione d'uso del tratto costiero e della valle si è via via consolidata nel tempo erodendo in maniera progressiva lo spazio pedecollinare con la collocazione del grande carbonile della centrale elettrica Enel sul versante orientale, cosicché la sommità del colle e porzioni limitate dei versanti nord-ovest e sud rimaste immobili sotto la proprietà demaniale, appaiono come un ultimo baluardo selvatico in un paesaggio marcatamente industriale. Questa architettura esercita il fascino proprio della rovina attraendo solo una minuscola porzione di pubblico di giocatori di Softair e giochi di ruolo dal vivo.

Concorre a questo stato di isolamento la difficoltà dell'accesso il quale avviene per mezzo della sola strada militare costruita a fine '800 (oggi via Pagliari), ad uso semi esclusivo del polo sportivo e che nel suo tratto terminale, superato quest'ultimo, prosegue sterrata.

A seguito della volontà manifestata dal Comune della Spezia di avviare il processo di recupero di questa fortificazione è stato iniziato un primo intervento conoscitivo della Batteria, con le operazioni di pulizia dalla vegetazione infestante cresciuta in maniera abbondante sopra tutta l'area e il successivo rilievo digitale completo, entrambe condotte tra giugno e ottobre 2019.

Il primo sopralluogo effettuato nella data 6 giugno 2018 aveva fatto emergere il degrado e lo stato di abbandono in cui versava il manufatto, le successive operazioni di pulizia eseguite a più riprese nel periodo estivo hanno permesso di ultimare la ricognizione e di comprendere l'effettivo stato di conservazione del manufatto e, nello specifico, la consistenza di quelle porzioni colpite dall'esplosione devastante avvenuta nel 1945. 
infatti a causa delle demolizioni operate dalle truppe tedesche in ritirata al termine della seconda guerra mondiale l'opera non è oggi completamente accessibile e presenta ampi crolli nelle pareti in prossimità dell'ingresso principale, così come nelle due caponiere di nord ovest e sud est.

\section{Rilievo digitale del complesso}

Il complesso di questa fortificazione presenta due aspetti inconsueti rispetto al comune intervento di rilievo: in primis si tratta di un complesso che ha subito un intervento distruttivo molto violento a seguito del quale la struttura è stata lungamente abbandonata e, in secondo luogo, si tratta di un edificio che ha appena ricevuto un intervento di pulizia dalla vegetazione, con ampie parti che si vanno a rivelare per la prima volta dopo un tempo molto lungo. A questo si aggiunge un aspetto consueto per il tipo di struttura: la questione del suolo e del territorio circostante.

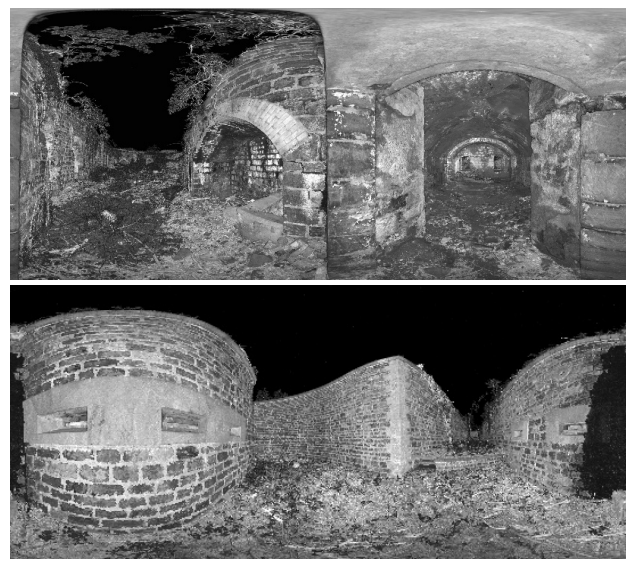

Figg. 2 e 3. Estratti di alcune delle scansioni effettuate nella fortezza durante la prima campagna di rilievo.

La fortificazione infatti risulta quasi interamente fusa con il territorio circostante, con aree verdi, spiazzi, strade e una rimodellazione del suolo complessiva che fa parte della struttura stessa, delle sue passate necessità difensive e di funzionamento. In questo senso, in base ai due punti iniziali, latori di una forte necessità di dettaglio, in modo da poter correttamente interpretare forme, volumi, spazi, ma anche patologie, spaccature e alterazioni, al contempo doveva essere as- solta la necessità di coprire abbastanza rapidamente le ampie aree in esterni, rendendo ben leggibile i caratteri del suolo dell'area circostante.

Gli elaborati richiesti per la documentazione e gestione di questo particolare patrimonio costruito erano configurati secondo un insieme di classici elaborati bidimensionali, con un livello di dettaglio sufficiente alla definizione di rappresentazioni in scala 1:20 ed atti ad entrare nel comune processo di gestione del bene, assieme alla specifica richiesta di definirne un modello tridimensionale digitale semplificato e parametrizzato, atto ad essere gestito come base di un processo H-BIM (Heritage Building Information Modeling) previsto utilizzando il software Graphisoft Archicad. Per operare efficacemente in questo senso le scelte sono state quelle di utilizzare una unità laser scanner 3D Zoller+Fröhlich $(\mathrm{Z}+\mathrm{F})$ Imager 5016 e basare le successive produzioni di elaborati sulla nuvola di punti allineata così generata. Nello specifico, l'unità laser scanner 3D scelta permette un ampio campo di presa, coprendo $360^{\circ}$ sull'orizzontale e $320^{\circ}$ sulla verticale, la sua tecnologia a "variazione di fase" (Phase-Shift) permette, con questo modello, di arrivare a misurare punti fino ad una distanza di $360 \mathrm{~m}$, in qualunque condizione di luce, con una accuratezza per ogni punto misurato di circa $2 \mathrm{~mm}$ a 10 $m$ di distanza.

La velocità di acquisizione, capace di arrivare fino a poco più di un milione di punti al secondo, permette agevolmente di completare una presa panoramica completa con la risoluzione di un punto battuto ogni centimetro a $10 \mathrm{~m}$ di distanza in un tempo di circa un minuto e mezzo a qualità standard (interpolazione di tre misurazioni consecutive dello stesso punto), ottimale per quasi tutti gli interni della fortezza e una scansione con la battitura di un punto ogni $6 \mathrm{~mm}$ a $10 \mathrm{~m}$ di distanza e con qualità alta (interpolazione di cinque misurazioni consecutive dello stesso punto) in circa sei minuti: una soluzione ottimale per buona parte delle aree esterne.

Tra le funzioni aggiuntive dello $\mathrm{Z}+\mathrm{F}$ Imager 5016 risulta apprezzabile la possibilità di ripresa a colori, effettuata con una fotocamera incorpo- 
rata capace di produrre un'immagine panoramica con risoluzione complessiva di 80 megapixels, la presenza di un sistema di illuminazione led integrato allo scanner permette la ripresa fotografica anche in situazioni di scarsa o nessuna illuminazione. In questo modo sono state eseguite, nell' arco di due sessioni operative un numero di 405 scansioni, a copertura completa di tutta la fortezza, avendo cura di coprire con limitato livello di occlusioni anche le aree che presentavano ancora vegetazione che le aree con crolli significativi e con passaggi o aperture particolarmente complicati da detriti e dissesti.

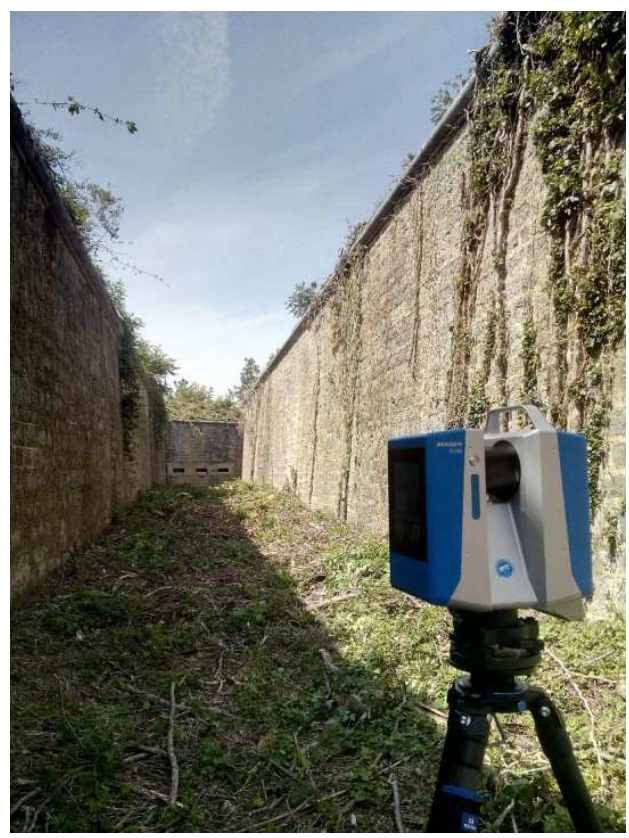

Fig. 3. Lo scanner Z+F Imager 5016 in fase di rilievo presso la Batteria Valdilocchi (Verdiani, 2019).

La campagna di rilievo è stata necessariamente suddivisa in due parti, una prima in Giugno, subito dopo il completamento della pulizia dalla vegetazione principale ed una successiva, in Ottobre, compiuta dopo il completamento della rimozione della vegetazione infestante ancora presente, come dei materiali tagliati e della spazzatura ammucchiata in anni di uso improprio del fabbricato.

La prima campagna ha quindi coperto tutti gli interni e tutta l'area perimetrale dell'edificio, fatta eccezione per circa $3 / 4$ del fronte principale crollato. Questa prima fase ha prodotto 232 scansioni ed ha richiesto tre giorni di operazioni. La seconda ha completato le operazioni sul fronte principale e ha portato al rilievo di tutta la copertura, dei suoi percorsi/vani, del cortile centrale e di alcuni spazi secondari precedentemente invasi da sporcizia, detriti o materiali da smaltire.

Questa seconda fase ha prodotto 172 scansioni ed ha richiesto due giorni di operazioni.

Tutte le scansioni, nella loro versione finale sono risultate contenere tra i 22 e i cinque milioni di punti, questo in base alla risoluzione di scansione adottata e all'effettiva presenza nello spazio di superfici scansionabili. In tutte le operazioni non sono stati utilizzati target specifici per facilitare il successivo processo di allineamento dell'intero insieme delle scansioni.

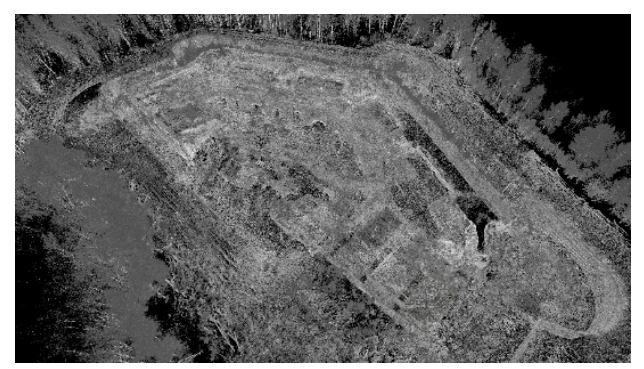

Fig. 4. Vista complessiva della nuvola di punti allineata (Verdiani, 2019).

La presenza di superfici murarie sempre ricche di dettagli, la morfologia caratterizzata da ampie aperture e spazi ristretti comunque con parti superiori alte e voltate e ricche di segni, macchie e elementi atti a facilitare l'allineamento per confronto geometrico, hanno permesso di ovviare all'applicazione di target piani o sferici accelerando sensibilmente il procedimento delle operazioni.

Ad integrazione delle operazioni di rilievo laser scanner 3D sono state eseguite numrose riprese fotografiche, sia utilizzando fotocamere digitali SLR con risoluzione tra i 20 e i 36,4 Megapixels che con fotocamere compatte con risoluzione di 16 Megapixels, l'archivio complessivo è stato redatto sia con fotografie documentative generali 
che relative ai dettagli, alle patologie più visibili, al quadro fessurativo, alle principali soluzioni costruttive e ai principali campioni murari, in modo da creare una documentazione completa utile a supportare appropriatamente le successive operazioni di trattamento del dato e la creazione di elaborati descrittivi chiari e dettagliati.

\subsection{Il trattamento del dato, nuvola di punti ed elaborati CAD 2D}

L'insieme dei file prodotti dallo Z+F Imager 5016, in formato ZFS, sono stati direttamente importati in Autodesk Recap Pro, dove sono stati filtrati per le principali anomalie di ripresa (essenzialmente per la presenza di punti sparsi, dovuti a riflessioni e/o presenza di luce parassita) e quindi allineati a comporre un unico modello composto da molteplici nuvole di punti e capace di descrivere dettagliatamente tutta la fortezza. Questo procedimento è stato svolto sia per l'insieme delle scansioni condotte in giugno che per quelle del completamento di ottobre.

I due gruppi di scansioni sono stati allineati in due gruppi separati e quindi composti in un unico modello. Le specifiche caratteristiche morfologiche della parte rilevata in ottobre non hanno infatti comportato limitazioni nella gestione del secondo allineamento, permettendo di ricomporre assieme tutte le parti in un nuovo modello indipendente dal primo.

I due gruppi sono stati quindi allineati a loro volta a comporre un unico modello complessivo di nuvola di punti. La procedura di messa a registro automatica ha funzionato in maniera ben efficace con il dato molto accurato prodotto dallo scanner e grazie ad una valida pianificazione delle postazioni di scansione, pensata già in fase di ripresa in maniera conveniente per permettere un positivo risultato di questa procedura automatizzata. Dalla nuvola di punti complessiva sono stati quindi estratti gli elaborati 2D in formato DWG, utilizzando l'apposita procedura di ridisegno in Autodesk Autocad. Questi disegni, trattati con un livello di dettaglio adeguato per la rappresentazione in scala 1:50, sono stati rapidamente tracciati ed hanno costituito una prima elaborazione "leggera" a documentazione dello stato della fortezza. Il dato nuvola di punti e l'insieme dei disegni DWG sono stati quindi portati come base per la successiva modellazione H-BIM.

\subsection{Il passaggio verso il modello H-BIM}

Una volta completate le operazioni di primo trattamento della nuvola di punti, si è proceduto a realizzare una versione "alleggerita" del modello complessivo, utilizzando l'apposita procedura supportata da Autodesk Recap è stata esportata una versione "RCP semplificata" in cui tutte le singole nuvole di punti sono state riunite secondo un'unica griglia di punti, ridefinita come regolare e con passo di $6 \mathrm{~mm}$.

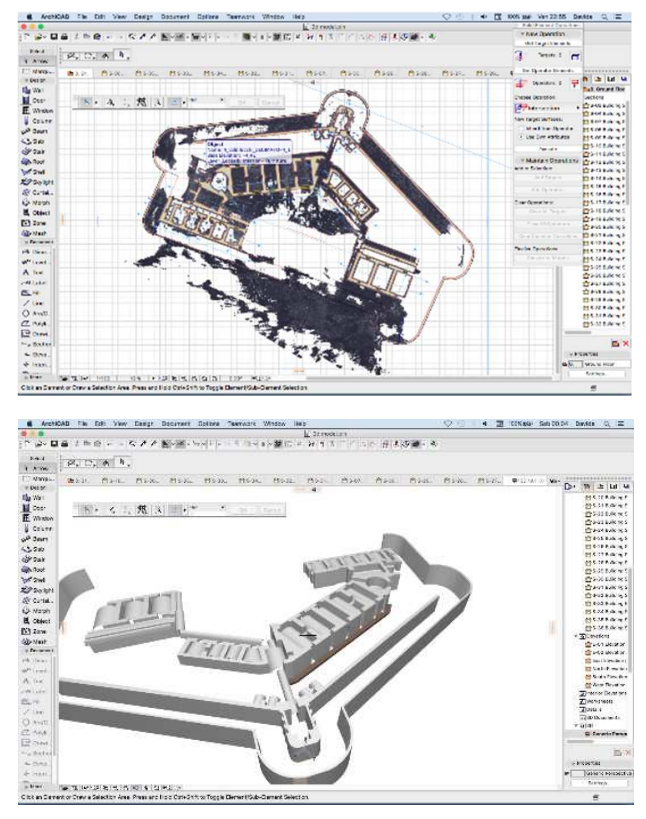

Figg. 5 e 6. Viste del modello per H-BIM in fase di sviluppo in Graphisoft Archicad (Sciannandrone, 2019).

Questa versione è stata quindi esportata in formato PTS, compatibile con l'impiego in Graphisoft Archicad per lo sviluppo del modello tridimensionale della base per H-BIM. Il termine $\mathrm{H}-\mathrm{BIM}$ indica ovviamente la versione "Heritage" di un sistema Building Information Modeling, ampiamente in uso nell'ambito della nuova progettazione e chiave delle operazioni architettoniche contemporanee di nuova progettazione e di intervento sull'esistente. Ad oggi l'impiego di questo tipo di modellazione per le architetture esistenti ha già una ampia gamma 
di esperienze, ma risulta ancora in parte "sperimentale" (Biagini, Arslan, 2018) e non pienamente codificata nelle soluzioni per il Patrimonio Costruito Storico (Camiz, Capparelli, 2019).

Le problematiche di trasposizione dal dato omogeneo della nuvola di punti a elementi parametrizzati ha richiesto una elaborazione attenta delle singole parti, dove l'aspetto massivo di molte parti architettoniche, ma pur riconducibile a componenti ben definite e "ricorrenti", ha aiutato non poco. Il modello così definito, seppur nella sua significativa semplificazione, è andato a costituire una base completa per la successiva analisi e considerazioni circa i necessari interventi da eseguire, un modello semplificato, ma "snello" e "pratico" in cui le specifiche caratteristiche di rappresentazione tridimensionali e la rapidità di gestione e aggiornamento portano alla piena centralità del modello digitale rispetto tutte le operazioni di valutazione e intervento.

La base conoscitiva e di rappresentazione digitale così sviluppata può essere impiegata per molteplici usi oltre la semplice documentazione, con la possibilità di ulteriori raffinamenti ed aggiornamenti al seguito dell'evolversi della struttura stessa a seguito dei prossimi interventi di restauro e riuso.

\section{Conclusioni}

Il processo di rilievo e restituzione digitale del modello diventano strumenti essenziali per riconoscere e ri-incontrare questa architettura dimenticata nel complesso territoriale e paesaggistico in cui si inserisce.

Il processo di rilievo digitale dunque non è stato concepito e proposto al solo fine di fornire una giusta base alla progettazione successiva che il Comune della Spezia ha richiesto per questo manufatto. Esso risponde all'obiettivo più ampio di restituire una conoscenza puntuale dell'architettura (e del contesto paesaggistico in cui si inserisce), del suo funzionamento e della sua storia consentendo attraverso il modello digitale e tutte le successive elaborazioni che da esso si potranno trarre, di far avvicinare le persone al patrimonio architettonico militare presente sul ter- ritorio, il quale oggi è pressoché misconosciuto e poco sfruttato sia sul piano dell'offerta culturale che su quella turistica e ricettiva, rispetto alla quale la città da anni sta implementando una politica proattiva ma poco creativa e lungimirante.

La batteria Valdilocchi dall'essere un'architettura alla fine di un'epoca, potrebbe attraverso questa prima fase di rigenerazione del suo immaginario storico, prefigurare finalmente nuovi usi compatibili, tornando a costituire per l'intera valle il volano per una rigenerazione sostenibile.

\section{Note}

Le attività di rilievo e documentazione della Batteria Valdilocchi si sono svolte nell'ambito della Convenzione di Ricerca conto terzi "Rilievo e Documentazione della Batteria Valdilocchi, La Spezia" tra il Dipartimento di Architettura dell'Università degli Studi di Firenze e il Comune di La Spezia.

Responsabile Scientifico: prof. Giorgio Verdiani. Hanno partecipato alle operazioni di rilievo: Giorgio Verdiani, Ludovica Marinaro, Andrea Landini, Davide Sciannandrone, Andrea Pasquali, Andrea Scippa.

Le restituzioni del dato da rilievo digitale sono state condotte da Giorgio Verdiani, Davide Sciannandrone, Andrea Landini.

Le strumentazioni in uso sono state rese disponibili dal sistema DIDALABS del Dipartimento di Architettura dell'Università degli Studi di Firenze. Si ringrazia l'associazione "Dalla parte dei Forti" della Spezia e nello specifico Stefano Danese, per il supporto tecnico e organizzativo dato al progetto; Il Comune della Spezia nella persona dell'assessore ai Lavori Pubblici Luca Piaggi, dell'ing. Gianluca Rinaldi (R.U.P. del progetto) e dell'intero Servizio Aree Verdi del Comune della Spezia. 


\section{Bibliography}

Agulhon, R.; Bassino, J.P.; Boniface, J.C. (1980). Protection integree du vigne, ITV-ACTA Issoudun Ed., Francia, pp. 79-148.

Ambrosi A.C. (1983). Straviario. tutte le vie grandi e piccole. tutte le piazze belle e brutte della Spezia vecchia e nuova, Cassa di Risparmio della Spezia, Stabilimento Tipografico Fabbiani.

Biagini, C.; Arslan, P. (2018). "Industrial Heritage in the historical neighbourhood: BIM strategies for urban regeneration”, in Giordano, A.; Huffman, K., eds., Disegnarecon Vol. 11, N. 21, Advanced Technologies for Historical Cities Visualization, University of L'Aquila.

Camiz, A.; Capparelli, F. (2019). "BIM documentation for architecture and archeology: the Shipwreck Museum in the Kyrenia Castle, Cyprus", in Conte, A.; Guida, A., eds., Re USO Matera PATRIMONIO IN DIVENIRE conoscere valorizzare abitare, proceedings of Reuso VII Convegno Internazionale sulla Documentazione, Conservazione e Recupero del Patrimonio Architettonico e sulla Tutela Paesaggistica, Dipartimento delle Culture Europee e del Mediterraneo Università degli Studi della Basilicata, Matera, Gangemi, pp. 1215-1226.

Danese, S. (2011). Difesa di una piazzaforte marittima: fortificazioni e artiglierie nel Golfo della Spezia dal 1860 al 1945, Autorità portuale della Spezia.

Di Grazia, S.; Marinaro L. (2015). "Fortifications and Landscape System. Geological and geomorphological resilience in the development of the La Spezia Gulf", in Rodriguez Navarro, P., ed., FORTMED 2015. Defensive Architecture of the Mediterranean. XV to XVIII Centuries, Editorial Universitat Politècnica de Valencia, Valencia, vol. I.

Faggioni, G. (2008). Fortificazioni in provincia della Spezia: 2000 anni di architettura militare, Ritter.

Marinaro, L.; Danese, S.; Carassale, S. (2016). "Palmaria. Un passato militare, un futuro Paesaggio. Prospettive per la valorizzazione paesaggistica e architettonica del sistema di fortificazioni dell'isola dopo la sdemanializzazione", in Verdiani, G., ed., FORTMED 2016. Defensive Architecture of the Mediterranean. XV to XVIII Centuries, DIDAPRESS, Firenze, vol. IV, pp. 365-372.

Marinaro, L.; Di Grazia, S. (2016). "La resilienza paesaggistica. Un approccio transdisciplinare alla progettazione", in Verdiani, G., ed., FORTMED 2016. Defensive Architecture of the Mediterranean. XV to XVIII Centuries, DIDAPRESS, Firenze, Vol. IV, pp. 341-348.

Marinaro, L.; Di Grazia, S. (2017). "Paesaggi Forti. Leggere le forme per riconfigurare i ruoli del sistema di fortificazioni dell' Arsenale Militare del Golfo della Spezia. Il caso di Marola”, in Gonzáles Avilés, Á., ed., FORTMED 2017. Defensive Architecture of the Mediterranean. XV to XVIII Centuries, Editorial Publicacion Universitat d'Alacant, Alacant, vol. VI, pp. 435-440

Marinaro, L.; Reitano, D.; Falqui, E. (2018). "Paesaggi sublimi. Un parco per valorizzare il patrimonio paesaggistico militare delle colline del Golfo della Spezia", in Marrotta, A.; Spallone, R., eds., FORTMED 2018. Defensive Architecture Of The Mediterranean, Politecnico di Torino, vol. IX, pp.1217-1222.

Marmori, F. (1976). Fortificazioni nel Golfo della Spezia: “architettura militare spezzina”, Stringa Editore. 